\title{
Doubly Special Relativity with light-cone deformation
}

\author{
A. Błaut* M. Daszkiewiczł and J. Kowalski-Glikman ${ }^{\ddagger}$ \\ Institute for Theoretical Physics \\ University of Wroctaw \\ Pl. Maxa Borna 9 \\ Pl-50-204 Wroctaw, Poland
}

November 5, 2018

\begin{abstract}
We propose a new Doubly Special Relativity theory based on the generalization of the $\kappa$-deformation of the Poincaré algebra acting along one of the null directions. We recall the quantum Hopf structure of such deformed Poincaré algebra and use it to derive the phase space commutation relations. As in the DSR based on the standard quantum $\kappa$-Poincaré algebra we find that the space time is non-commutative. We investigate the fate of the properties of Special Relativity in the null basis: the split of the algebra of Lorentz and momentum generators into kinematical and dynamical parts, the action of the kinematical boost $\mathrm{M}^{+-}$, and the emergence of the two dimensional Galilean symmetry.
\end{abstract}

\footnotetext{
*e-mail address ablaut@ift.uni.wroc.pl

$\dagger$ e-mail address marcin@ift.uni.wroc.pl

${ }^{\ddagger}$ e-mail address jurekk@ift.uni.wroc.pl

§Partially supported by the KBN grant 5PO3B05620
} 


\section{Introduction}

The fate of Lorentz symmetry at high energies close to the Planck scale is recently attracting growing interest. The reason for this is at least twofold. First, since the classical Lorentz symmetry group is non-compact we have only limited access to experimental verification of this symmetry. Second, the observed anomalies in the ultra-high energy cosmic rays and $\mathrm{TeV}$ photons [1] might indicate that relativistic kinematics may deviate from the standard Special Relativistic one when relevant energies becomes close to the Planck scale.

There are two major approaches to the "Planck scale phenomenology" (see [2] and [3] for reviews and extesive list of references). In the first one assumes that Lorentz symmetry becomes broken close to the Planck scale, so that effectively we have to do with some preferred frame, usually associated with the cosmic frame. In another approach, dubbed Doubly Special Relativity [7, 6], one assumes that Lorentz (and even Poincaré) symmetry is still present, but becomes deformed in the high energy regime. This approach borrows a lot from previous investigations on quantum deformation of Poincaré Hopf algebra, undertaken in 1990s (see e.g., [4], [5]).

Presently two Doubly Special Relativity models have received most attention $^{1}$. In the first, called DSR1 formulated in [8] and 9] the modified dispersion relation takes the form

$$
m^{2}=\left(2 \kappa \sinh \left(\frac{P_{0}}{2 \kappa}\right)\right)^{2}-\vec{P}^{2} e^{P_{0} / \kappa}
$$

while in the DSR2, proposed in [10] it reads

$$
m^{2}=\frac{P_{0}^{2}-\vec{P}^{2}}{\left(1-\frac{P_{0}}{\kappa}\right)^{2}}
$$

Let us note that the deformation in the dispersion relations (11), (2) act in the "energy" direction. This is a general feature of all DSR theories considered so far, and a natural question arises as to whether it is possible to construct DSR theories with different kind of deformations. For example is it possible to construct a DSR theory with deformation along "light cone" corresponding

\footnotetext{
${ }^{1}$ As shown in the series of papers [1], 12, 13] there are in fact infinitely many DSR theories.
} 
to the front form dynamics in the famous classification of Dirac [14] (see also e.g. 15], 16] for extensive up-to-date discussion and list of references.) The answer to this question happens to be affirmative [17, [18, [19], 20] and we devote this note to investigate most fundamental properties of such theories.

Our motivations to investigate light-cone DSR are manyfold. First, such DSR theories differ in many respects from the theories analyzed so far and therefore are of interest on its own. Moreover the standard light-cone dynamics has a number of distinctive features which, in the deformed case may shed some light on generic structure of Doubly Special Relativity. Among them one should mention the rational relation between energy and momentum on-

shell $P_{-}=\frac{\vec{P}^{2}+m^{2}}{P_{+}}$as compared to $P_{0}=\sqrt{\vec{P}^{2}+m^{2}}$ of standard "instant" formulation of Special relativity, special structure of boosts of which one is a kinematical operator acting along the constant "light-cone time" surface, and the emergence of Galilean symmetry as a subgroup of the whole Poincaré algebra (and not the reduction of the latter, as in the standard case.) Last but not least, it were intuitions from the light cone dynamics that lead Susskind [21] to formulate his version of the holographic principle, so one may hope that proper understanding of the deformed light-cone dynamics may lead to better understanding of this principle.

\section{DSR phase space in light-cone coordinates}

Our starting point of the construction of the light-cone DSR theory will be the light-cone quantum $\kappa$-Poincaré algebra. This kind of algebras have been investigated first in [17, whose construction has been incorporated into the generalized $\kappa$-Poincaré framework in [18] and [19]. As usual in the light-cone formulation momenta has components $P_{+}, P_{-}$along the light-cone and the transverse ones $P_{i}{ }^{2}$. The metric tensor in such parameterization has the form

$$
g_{\mu \nu}=g^{\mu \nu}=\left(\begin{array}{cccc}
0 & 1 & 0 & 0 \\
1 & 0 & 0 & 0 \\
0 & 0 & -1 & 0 \\
0 & 0 & 0 & -1
\end{array}\right)
$$

\footnotetext{
${ }^{2}$ In what follows we will use the following index convention: Greek indices run through $+,-, 1,2$, small Latin indices denote transverse directions 1,2 , while capital ones run through $-, 1,2$.
} 
The action of Lorentz generators $M^{\mu \nu}$ satisfies the standard undeformed algebra

$$
\left[M^{\mu \nu}, M^{\lambda \sigma}\right]=i\left(g^{\mu \sigma} M^{\nu \lambda}-g^{\nu \sigma} M^{\mu \lambda}+g^{\nu \lambda} M^{\mu \sigma}-g^{\mu \lambda} M^{\nu \sigma}\right),
$$

and the momenta commute

$$
\left[P_{\mu}, P_{\nu}\right]=0 .
$$

As usual in DSR the action of Lorentz generators on momenta is deformed with the scale of deformation given by the parameter $\kappa$ of dimension of mass.

$$
\begin{aligned}
{\left[M^{i j}, P_{+}\right] } & =\left[M^{i-}, P_{+}\right]=0 \\
{\left[M^{i+}, P_{+}\right] } & =i P^{i} \\
{\left[M^{-+}, P_{+}\right] } & =i \kappa\left(1-e^{-P_{+} / \kappa}\right) \\
{\left[M^{i j}, P_{-}\right] } & =0 \\
{\left[M^{i+}, P_{-}\right] } & =-\frac{i}{\kappa} P^{i} P_{-} \\
{\left[M^{i-}, P_{-}\right] } & =i P^{i} \\
{\left[M^{-+}, P_{-}\right] } & =-i P_{-}-\frac{i}{2 \kappa} \vec{P}^{2} \\
{\left[M^{i j}, P_{k}\right] } & =i\left(\delta^{j}{ }_{k} P^{i}-\delta^{i}{ }_{k} P^{j}\right) \\
{\left[M^{i+}, P_{k}\right] } & =-i \delta^{i}{ }_{k}\left(P_{-} e^{-P_{+} / \kappa}+\frac{1}{2 \kappa} \vec{P}^{2}\right)-\frac{i}{\kappa} P^{i} P_{k} \\
{\left[M^{i-}, P_{k}\right] } & =-i \kappa \delta^{i}{ }_{k}\left(1-e^{-P_{+} / \kappa}\right) \\
{\left[M^{-+}, P_{k}\right] } & =-i P_{k}\left(1-e^{-P_{+} / \kappa}\right),
\end{aligned}
$$

where we define $\vec{P}^{2}=P_{1}^{2}+P_{2}^{2}$. The Casimir (dispersion relation) for this algebra reads

$$
m^{2}=4 \kappa P_{-} e^{P_{+} / 2 \kappa} \sinh \frac{P_{+}}{2 \kappa}-\left(P_{1}^{2}+P_{2}^{2}\right) e^{P_{+} / \kappa}
$$

which, of course, reduces (as well as the algebra above) to the standard relation of the null-frame Special Relativity in the $\kappa \rightarrow \infty$ limit. Note that in the large $P_{+}$limit the dispersion relation becomes in the leading order

$$
\vec{P}^{2}=2 \kappa P_{-}
$$


independently of mass, as compared with the relation

$$
\vec{P}^{2}+m^{2}=2 P_{+} P_{-}
$$

of the undeformed case.

The fact that the algebra (5) can be extended to a quantum Hopf algebra makes it possible to use the co-product of the latter to construct the commutators algebra of the whole the phase space of the system. This procedure consists first of defining pairing between Lorentz generators and momenta position $X^{\mu}$. To do that one takes the Hopf algebra co-products

$$
\begin{aligned}
\Delta\left(P_{+}\right) & =1 \otimes P_{+}+P_{+} \otimes 1 \\
\Delta\left(P_{I}\right) & =P_{I} \otimes e^{-P_{+} / \kappa}+1 \otimes P_{I} \\
\Delta\left(M^{I J}\right) & =1 \otimes M^{I J}+M^{I J} \otimes 1 \\
\Delta\left(M^{I+}\right) & =M^{I+} \otimes e^{-P_{+} / \kappa}+1 \otimes M^{I+}-\frac{1}{\kappa} M^{I J} \otimes P_{J} \\
\Delta\left(X^{\mu}\right) & =\Lambda^{\mu}{ }_{\nu} \otimes X^{\nu}+X^{\mu} \otimes 1 \\
\Delta\left(\Lambda^{\mu}{ }_{\nu}\right) & =\Lambda^{\mu}{ }_{\rho} \otimes \Lambda^{\rho}{ }_{\nu}
\end{aligned}
$$

and then defines the pairing as follows

$$
\begin{aligned}
<P_{\mu}, X^{\nu}> & =i \delta_{\mu}^{\nu} \\
<\Lambda^{\mu}{ }_{\nu}, M^{\alpha \beta}> & =i\left(g^{\alpha \mu} \delta_{\nu}^{\beta}-g^{\beta \mu} \delta_{\nu}^{\alpha}\right) \\
<\Lambda^{\mu}{ }_{\nu}, 1> & =\delta_{\nu}^{\mu}
\end{aligned}
$$

In the final step one uses eqs. (92) to derive relevant commutators according to the general formula

$$
[\mathcal{Q}, \mathcal{R}]=\mathcal{R}_{(1)}<\mathcal{Q}_{(1)}, \mathcal{R}_{(2)}>\mathcal{Q}_{(2)}-\mathcal{R} \mathcal{Q},
$$

where we used a natural notation for co-product $\Delta \mathcal{R}=\sum \mathcal{R}_{(1)} \otimes \mathcal{R}_{(2)}$. The resulting non-vanishing phase space commutators read

$$
\begin{gathered}
{\left[X^{+}, X^{i}\right]=\frac{i}{\kappa} X^{i} \quad\left[X^{+}, X^{-}\right]=\frac{i}{\kappa} X^{-}} \\
{\left[P_{i}, X^{j}\right]=-i \delta_{i}^{j} \quad\left[P_{i}, X^{+}\right]=\frac{i}{\kappa} P_{i}} \\
{\left[P_{-}, X^{-}\right]=-i \quad\left[P_{-}, X^{+}\right]=\frac{i}{\kappa} P_{-} \quad\left[P_{+}, X^{+}\right]=-i}
\end{gathered}
$$


The transformation of positions under action of Lorentz generators are given by

$$
\begin{gathered}
{\left[X^{\mu}, M^{K+}\right]=i\left(g^{K \mu} X^{+}-g^{+\mu} X^{K}\right)-\frac{i}{\kappa}\left(\delta_{+}^{\mu} M^{K+}+\delta_{L}^{\mu} M^{K L}\right)} \\
{\left[X^{\mu}, M^{K L}\right]=i\left(g^{K \mu} X^{L}-g^{L \mu} X^{K}\right)}
\end{gathered}
$$

To conclude this technical section stressing that the phase space algebra constructed above can be altered by redefinition of momenta of the form

$$
\begin{aligned}
& P_{+} \rightarrow \mathcal{P}_{+}\left(P_{+}, P_{-}, \vec{P}^{2}\right) \\
& P_{-} \rightarrow \mathcal{P}_{-}\left(P_{+}, P_{-}, \vec{P}^{2}\right) \\
& P_{-} \rightarrow \mathcal{P}_{i}\left(P_{+}, P_{-}, \vec{P}^{2}, P_{i}\right)=A\left(P_{+}, P_{-}, \vec{P}^{2}\right) P_{i}
\end{aligned}
$$

This redefinition is restricted only by the natural requirement that in the $\kappa \rightarrow \infty$ limit $P_{\mu}=\mathcal{P}_{\mu}$, which means that in this limit all the light-cone DSR theories reduce to Special Relativity in the null frame.

\section{Properties of the phase space algebra}

Let us now discuss the properties of the deformed phase space derived in the previous section. We will focus our attention on the fate of three distinguishing properties of Special Relativity formulated in null frame: split of the Poincaré generators into seven kinematical and three dynamical ones, the simple action of the boost in the third direction $\mathrm{M}^{-+}$and the emergence of the Galilei algebra as a subalgebra of the Poinceré light-cone algebra. Before doing that, let us briefly describe these three features.

In his seminal paper [14] Dirac considered the possible forms of initial surfaces for relativistic dynamics. Given an initial surface it is important to know which Poincaré symmetry generators act along the surface (these are called kinematical) and which in the orthogonal direction (since these generators change the value of the coordinate orthogonal to the surface - the time variable - they are called dynamical.) In the case of constant time initial surface (instant form Special Relativity), it is well known that there are six kinematical (spacial momenta and rotations) generators and four dynamical ones (boosts and energy generator). In the null frame Special Relativity, when the initial surface is null it turns out that there are seven kinematical 
generators (it can be shown that this is the maximal possible number of them) and three kinematical ones.

The generator that is dynamical in the instant form, and becomes kinematical in the null form is the boost along the third axis $M^{+-}$. Moreover this generator acts as a mere rescaling of positions and momenta, to wit

$$
X^{+} \rightarrow e^{\xi} X^{+}, \quad X^{-} \rightarrow e^{-\xi} X^{-}, \quad P^{+} \rightarrow e^{\xi} P^{+}, \quad P^{-} \rightarrow e^{-\xi} P^{-},
$$

where $\xi$ is the boost parameter. Clearly these transformation leaves the surface $X^{+}=0$ (or $X^{-}=0$ ) invariant, so indeed $M^{+-}$is kinematical.

The last feature of the null frame form is that the subalgebra of the whole Poincaré algebra of the longitudinal symmetry generators is isomorphic to the Galilei [22] algebra with the following identification: $P^{i}$ correspond to Galilei momenta, $M^{i j}$ to rotations, $P^{-}$to Hamiltonian, while $P^{+}$is a central charge of the subalgebra, corresponding to the Galilei mass (see below.) This situation should be compared with the instant form Special Relativity, where the Galilei algebra arises only as an appropriate limit of the Poincaré algebra.

To conclude these introductory remarks, let us note that contrary to the Special Relativistic case there is no $+\leftrightarrow-$ symmetry between coordinates $X^{+}$and $X^{-}$(and momenta $P^{+}$and $P^{-}$), and we must therefore consider the cases of null surfaces $X^{+}=0$ and $X^{-}=0$ separately. As it will turn out these surfaces have very different properties.

\section{Kinematical generators}

In the first case, in order to find out which generators are kinematical and which dynamical we must consider generators of the stability group of the surface $X^{+}=0$. Consider first the algebra (14), (15). We have

$$
\begin{gathered}
{\left[X^{+}, M^{K+}\right]=-\frac{i}{\kappa} M^{K+}} \\
{\left[X^{+}, M^{K L}\right]=i\left(g^{K+} X^{L}-g^{L+} X^{K}\right)}
\end{gathered}
$$

From (18) we learn that $M^{i j}$ is kinematical, as in the standard case. However $M^{+i}$ and $M^{+-}$have non-vanishing commutator (17) and therefore are not kinematical. The inspection of the algebra (12), (13) shows that also the momenta $P_{i}, P_{-}$are not kinematical. 
Consider therefore the null surface $X^{-}=0$. The algebra (14), (15) reads in this case

$$
\begin{gathered}
{\left[X^{-}, M^{K+}\right]=-i X^{K}-\frac{i}{\kappa} M^{K-}} \\
{\left[X^{-}, M^{K L}\right]=0}
\end{gathered}
$$

We see that now the kinematical generators are $M^{i j}, M^{-+}, M^{i-}$. From (12), (13) $)$ we see that also the momenta $P_{i}, P_{-}$are kinematical. Therefore in this case we have as many kinematical generators as in Special Relativity.

To conclude, for the initial surface $X^{+}=0$ the number of kinematical generators is small, but the coordinates on the surface are commutative. It is light-cone time $X^{+}$that is non-commutative, and the light-cone energy can be associated with the $P_{+}$generator. For the initial surface $X^{-}=0$ the number of kinematical generators is exactly equal to the corresponding number in Special Relativity, but, the coordinates on the surface are not commutative. In this case $X^{-}$and $P_{-}$play a role of time and energy, respectively.

\section{The $M^{-+}$boost}

To see what is the behavior of the $M^{-+}$boost for deformed algebra, let us first consider the commutators with momenta (5) and infer from them

$$
\begin{aligned}
& \frac{d P_{+}}{d \xi}=\kappa\left(1-e^{-P_{+} / \kappa}\right) \\
& \frac{d P_{-}}{d \xi}=-P_{-}-\frac{1}{2 \kappa} \vec{P}^{2} \\
& \frac{d P_{k}}{d \xi}=-P_{k}\left(1-e^{-P_{+} / \kappa}\right)
\end{aligned}
$$

where $\xi$ is the rapidity parameter. The solution of these equations with the initial condition $P_{\mu}(0)=\pi_{\mu}$ reads

$$
\begin{gathered}
P_{+}(\xi)=\kappa \log \left[1+C e^{\xi}\right], \quad P_{i}(\xi)=\frac{(C+1) \pi_{i}}{1+C e^{\xi}} \\
P_{-}(\xi)=e^{-\xi}\left[\pi_{-}-\frac{(C+1) \vec{\pi}^{2}}{2 \kappa C}+\frac{(C+1)^{2} \vec{\pi}^{2}}{2 \kappa C\left(1+C e^{\xi}\right)}\right]
\end{gathered}
$$

where $C=e^{\pi_{+} / \kappa}-1$. This last equation can be simplified if one uses the dispersion relation satisfied by the initial values of momenta

$$
4 \kappa \pi_{-} e^{\pi_{+} / 2 \kappa} \sinh \frac{\pi_{+}}{2 \kappa}-\vec{\pi}^{2} e^{\pi_{+} / \kappa}=m^{2} .
$$


One gets

$$
P_{-}(\xi)=e^{-\xi}\left[\frac{m^{2}}{2 \kappa C}+\frac{(C+1)^{2} \vec{\pi}^{2}}{2 \kappa C\left(1+C e^{\xi}\right)}\right]
$$

Expanding to the lowest order in $\kappa$ we get the standard relations

$$
P_{+}(\xi) \sim \pi_{+} e^{\xi}, \quad P_{-}(\xi) \sim \pi_{-} e^{-\xi}, \quad P_{i}(\xi) \sim 0
$$

as it should be. The expansion for large boosts $\xi \rightarrow \infty$ gives instead (in the massless case, for simplicity)

$$
P_{+}(\xi) \sim \xi, \quad P_{i}(\xi) \sim e^{-\xi}, \quad P_{-}(\xi) \sim e^{-2 \xi} .
$$

This expansion is consistent with relation eq. (77). Note that while the behavior of $P_{-}(\xi)$ is similar to the standard one, $P_{+}(\xi)$ grows with $\xi$ much slower than in Special Relativity.

\section{Galilean symmetry}

As mentioned above, the last interesting feature of the Special Relativity in the null frame is that there exist two subalgebras of the full Poincaré algebra that are isomorphic to the algebra of the two-dimensional Galilei group. These subalgebras consist of the rotation $M^{i j}$, Galilean boosts $G_{ \pm}^{i}=M^{ \pm i}$, and $P_{\mu}$ and read

$$
\begin{aligned}
{\left[M^{i j}, G_{ \pm}^{k}\right] } & =i\left(g^{i k} G_{ \pm}^{j}-g^{j k} G_{ \pm}^{i}\right) \\
{\left[M^{i j}, P_{k}\right] } & =i\left(\delta^{j}{ }_{k} P^{i}-\delta^{i}{ }_{k} P^{j}\right) \\
{\left[G_{ \pm}^{i}, P_{+}\right] } & =-i \delta_{+}^{ \pm} P^{i} \\
{\left[G_{ \pm}^{i}, P_{-}\right] } & =-i \delta_{-}^{ \pm} P^{i} \\
{\left[G_{ \pm}^{i}, P_{k}\right] } & =-i \delta^{i}{ }_{k} P_{\mp}
\end{aligned}
$$

Note that in the '-' case $P_{+}$is a central charge of the algebra and can be therefore identified with (twice) the Galilean mass, while $P_{-}$is the Hamiltonian (in the ' + ' case the mass is identified with $P_{-}$, and Hamiltonian with $P_{+}$.)

Inspection of the algebra (5) shows that in the deformed algebra this doubly structure is lost. Indeed, in this case only $P_{+}$can be central charge, 
as it commutes with $G_{-}^{i}$. In this way we obtain the deformed Galilei algebra, being a subalgebra of the deformed Poincaré algebra (5), to wit

$$
\begin{aligned}
{\left[M^{i j}, G^{k}\right] } & =i\left(g^{i k} G_{ \pm}^{j}-g^{j k} G_{ \pm}^{i}\right) \\
{\left[M^{i j}, P_{k}\right] } & =i\left(\delta_{k}^{j} P^{i}-\delta^{i}{ }_{k} P^{j}\right) \\
{\left[G^{i}, P_{-}\right] } & =-i \delta_{-}^{ \pm} P^{i} \\
{\left[G^{i}, P_{k}\right] } & =i \kappa \delta_{k}^{i}\left(1-e^{-P_{+} / \kappa}\right)
\end{aligned}
$$

where we used the notation $G^{i}=G_{-}^{i}$. Since $P_{-}=P^{+}$is the Hamiltonian, it corresponds to the initial null surface defined by $X^{-}=0$, because in this case $X^{-}$takes a role of time.

\section{Conclusions}

In this paper we investigated basic properties of a new Doubly Special Relativity in which the deformation acted along the light cone. This theory differs in many aspects from the DSR theories considered so far, and in our opinion deserves farther studies.

However it is our view that the final judgment of which theory is correct is provided by its experimental verification. In the case of the DSR theories one of the few experiments that can be performed in a near future are "time of flight experiments", i.e., measurements of dependence of the speed of light on momentum carried by photons (see, e.g., [23] and references therein.) Therefore, it is important to investigate in details the issue of velocity in DSR theories. We will address this problem in a future publication.

\section{Acknowledgement}

We would like to thank J. Lukierski for discussion and bringing the paper 20] to our attention.

\section{References}

[1] G. Amelino-Camelia and T. Piran, "Planck-scale deformation of Lorentz symmetry as a solution to the UHECR and the TeV-gamma paradoxes," Phys. Rev. D 64 (2001) 036005 arXiv:astro-ph/0008107; 
G. Amelino-Camelia, J. Lukierski and A. Nowicki, "Absorption Of Tev Photons And Kappa-Deformed Poincare Algebra," Czech. J. Phys. 51 (2001) 1247 S. Sarkar, Mod. Phys. Lett. A 17, 1025 (2002), gr-qc/0204092, R. Aloisio, P. Blasi, A. Galante, P.L. Ghia, A.F. Grillo, astro-ph/0205271.

[2] G. Amelino-Camelia, "Quantum space-time: Deformed symmetries versus broken symmetries," arXiv:gr-qc/0201012

[3] G. Amelino-Camelia, "On the fate of Lorentz symmetry in loop quantum gravity and noncommutative spacetimes," arXiv:gr-qc/0205125.

[4] J. Lukierski, H. Ruegg, A. Nowicki and V. N. Tolstoi, "Q deformation of Poincare algebra," Phys. Lett. B 264 (1991) 331.

[5] S. Majid and H. Ruegg, "Bicrossproduct structure of kappa Poincare group and noncommutative geometry," Phys. Lett. B 334 (1994) 348 arXiv:hep-th/9405107.

[6] G. Amelino-Camelia, "Testable scenario for relativity with minimumlength," Phys. Lett. B 510, 255 (2001) arXiv:hep-th/0012238.

[7] G. Amelino-Camelia, "Relativity in space-times with short-distance structure governed by an observer-independent (Planckian) length scale," Int. J. Mod. Phys. D 11, 35 (2002) arXiv:gr-qc/0012051.

[8] J. Kowalski-Glikman, "Observer independent quantum of mass," Phys. Lett. A 286, 391 (2001) arXiv:hep-th/0102098.

[9] N. R. Bruno, G. Amelino-Camelia and J. Kowalski-Glikman, "Deformed boost transformations that saturate at the Planck scale," Phys. Lett. B 522, 133 (2001) arXiv:hep-th/0107039.

[10] J. Magueijo and L. Smolin, "Lorentz invariance with an invariant energy scale," Phys. Rev. Lett. 88 (2002) 190403 arXiv:hep-th/0112090.

[11] J. Kowalski-Glikman, "De Sitter space as an arena for doubly special relativity," Phys. Lett. B 547, 291 (2002) arXiv:hep-th/0207279.

[12] J. Kowalski-Glikman and S. Nowak, "Non-commutative space-time of doubly special relativity theories," arXiv:hep-th/0204245. 
[13] J. Kowalski-Glikman and S. Nowak, "Doubly special relativity theories as different bases of kappa-Poincare algebra," Phys. Lett. B 539, 126 (2002) arXiv:hep-th/0203040.

[14] P.A.M. Dirac, "Forms of relativistic dynamics", Rev. Mod. Phys. 21, 392 (1949).

[15] H. Leutwyler and J. Stern, "Relativistic dynamics on a null plane", Ann. Phys. 112, 94 (1978).

[16] T. Heinzl, "Light-cone quantization: foundations and application", arXiv:hep-th/0008096

[17] A. Ballesteros, F. J. Herranz, M. A. del Olmo and M. Santander, "A New 'Null Plane' Quantum Poincare Algebra," Phys. Lett. B 351 (1995) 137.

[18] P. Kosinski and P. Maslanka, "The $\kappa$-Weyl group and its algebra", in From field theory to quantum groups, B. Jancewicz and J. Sobczyk eds., World Scientific 1996 arXiv:q-alg/9512018.

[19] K. Przanowski, "The Hopf algebra isomorphism between $\kappa$-Poincaré algebra in the case $g^{00}=0$ and the "null plane" qunatum Poincaré algebra", Acta Phys. Polon. 28, (1997) 1635 arXiv:q-alg/9611025.

[20] J. Lukierski, V. Lyakhovsky and M. Mozrzymas, " $\kappa$-deformations of D $=4$ Weyl and conformal symmetries," Phys. Lett. B 538 (2002) 375 arXiv:hep-th/0203182.

[21] L. Susskind, "The World as a hologram," J. Math. Phys. 36 (1995) 6377 arXiv:hep-th/9409089.

[22] L. Susskind, "Model of self-induced strong interactions", Phys. Rev. 165 (1968) 1535; K. Bardakci and M.B. Halpern, "Theories at infinite momentum", Phys. Rev. 176 (1968) 1686.

[23] G. Amelino-Camelia, "Are we at the dawn of quantum-gravity phenomenology?," Lect. Notes Phys. 541, 1 (2000) arXiv:gr-qc/9910089. 